\title{
Flamand, hollandais, néerlandais... Badumes, standards et norme(s) dans les Bas-Pays
}

Flemish, Hollandish, Dutch, etc.: familiar registers, standard registers and norme(s) in the Low Countries

Jean Le Dû

\section{OpenEdition}

\section{Journals}

Édition électronique

URL : http://journals.openedition.org//bl/510

DOI : $10.4000 / / \mathrm{lbl} .510$

ISSN : 2727-9383

\section{Éditeur}

Université de Bretagne Occidentale - UBO

\section{Édition imprimée}

Date de publication : 30 décembre 2019

Pagination : 77-99

ISBN : 979-10-92331-49-3

ISSN : 1270-2412

\section{Référence électronique}

Jean Le Dû, « Flamand, hollandais, néerlandais... Badumes, standards et norme(s) dans les Bas-

Pays », La Bretagne Linguistique [En ligne], 23 | 2019, mis en ligne le 01 mai 2020, consulté le 03 mai 2021. URL : http://journals.openedition.org//bl/510 ; DOI : https://doi.org/10.4000//bl.510

La Bretagne Linguistique est mise à disposition selon les termes de la Licence Creative Commons Attribution 4.0 International. 


\title{
Flamand, hollandais, néerlandais... Badumes, standards et norme(s) dans les Bas-Pays
}

\author{
JEAN LE DÛ*
}

Tangue néerlandaise est une grande inconnue pour la plupart des —Français. Ce qui est d'autant plus étrange qu'il s'agit de la langue de pays voisins, et même d'une partie du territoire national. On entend souvent dire que les Flamands parlent le flamand et les hollandais le hollandais, mais sans connaitre les liens qui les unissent. On a la vague impression qu'il s'agit d'un «mélange d'allemand et d'anglais».

Mon ami Armel Wynants, professeur de néerlandais à l'université de Liège, m'a raconté le désappointement de ses élèves francophones quand ils essayaient de mettre en pratique leurs connaissances dans la Flandre voisine, à une demi-heure de voiture. À peine avaient-ils prononcé quelques phrases que leur interlocuteur les félicitait : «Toi, tu parles le néerlandais mieux que nous !», et la conversation se poursuivait... en français. Le même phénomène se produit en Basse-Bretagne, quand les apprentis bretonnants se voient propulsés au rang de connaisseurs du "vrai» breton par des locuteurs natifs, qui font ensuite semblant d'être indignes de converser en cette langue prestigieuse...

\section{Langue, dialecte, patois?}

Le sens commun considère que les langues sont des objets finis aux contours bien tranchés. Si on est bien obligé de reconnaître l'existence de variétés régionales, qu'on les nomme dialectes ou patois, on les voit comme de simples déformations de la «vraie» langue. Mais qu'est-ce

* Professeur émérite de celtique et breton, CRBC (EA 4451), UBO, Brest. 
donc qu'une langue ? Yves Le Berre et moi-même nous sommes posés cette question, confrontés il y a une quarantaine d'années à la mise en place d'un enseignement approfondi de la langue bretonne dans le cadre de la licence nouvellement créée. Le breton, en effet, ne se présente que sous la forme de «dialectes» variés et de graphies diverses. De tâtonnement en tâtonnement, d'un congrès de sociolinguistique à l'autre, nous avons élaboré un système par paliers qui nous semble rendre compte de la situation de la langue bretonne dans son histoire et son environnement social ${ }^{1}$. Nous en sommes venus à considérer qu'il n'existe pas de langues naturelles opposables à des langues culturelles; que la communication interindividuelle est toujours régie par des institutions, terme auquel nous donnons un sens élargi, allant du marché local à l'État national en passant par tous les stades intermédiaires du développement social. Ces notions sont-elles valides pour d'autres langues ? C'est ce que je vais tenter de vérifier dans cette courte présentation de la langue néerlandaise ${ }^{2}$.

\section{Les parlers de tradition orale immémoriale : les badumes}

Pendant l'hiver de 1954, j'ai tenté de mettre à l'épreuve le peu de néerlandais que j'avais appris avec la méthode Assimil. Je me suis adressé au mousse d'un des nombreux drifters - ou harenguiers hollandais qui faisaient escale tous les week-ends d'hiver dans ma ville natale de Dieppe. Surpris de m'entendre, il m’a déclaré : «Je spreekt goed Scheveningens!» 'Tu parles bien le Scheveningois !', c'est-à-dire le parler de Scheveningen, son port d'origine. Ce que son camarade a aussitôt rectifié : «mais non, c'est du hollandais !»... On touche là aux deux extrémités d'un continuum menant du parler local, encore d'usage commun à cette époque en Hollande, à un ensemble linguistique plus vaste auquel on a conscience d'appartenir. Ces mêmes gamins jouaient et bavardaient sans problèmes apparents de communication avec leurs

1. Jean LE Dû et Yves LE BerRe, Métamorphoses, Trente ans de sociolinguistique à Brest (1984-2014), Brest, Centre de Recherche Bretonne et Celtique, 2019.

2. Le CRBC a déjà publié des articles sur cette langue : Kas DEPREZ, "Soldats du néerlandais ", La Bretagne linguistique, $\mathrm{n}^{\circ}$ 10, actes du colloque Badume-StandardNorme : Le double jeu de la langue, Université de Bretagne Occidentale, Brest, CRBC, 1995, p. 189-215. Armel WYNANTS, "Les Fourons : Platt-Dütsch, wallon, néerlandais, allemand, français", La Bretagne linguistique, n 10, op. cit., p. 217-229. 
homologues allemands de Bremerhaven quand leurs bateaux étaient amarrés bord à bord.

Les badumes - mot que nous avons créé à partir du breton $b a$ dumañ "chez nous» - sont les idiomes correspondant à ce que nous nommons des "institutions d'usage», aux règles justifiées uniquement par la tradition orale, en vigueur dans un espace très restreint. Ils sont l'objet de constants discours évaluatifs : "Chez nous on dit... mais les autres disent». Toujours restreints à l'oralité, ils constituent l'objet d'étude des géolinguistes, qui les retranscrivent sur des cartes d'atlas. Leurs usagers les nomment "ce qu'on parle chez nous", ou, alternativement, les désignent du nom de leur localité, comme le faisait mon jeune mousse hollandais.

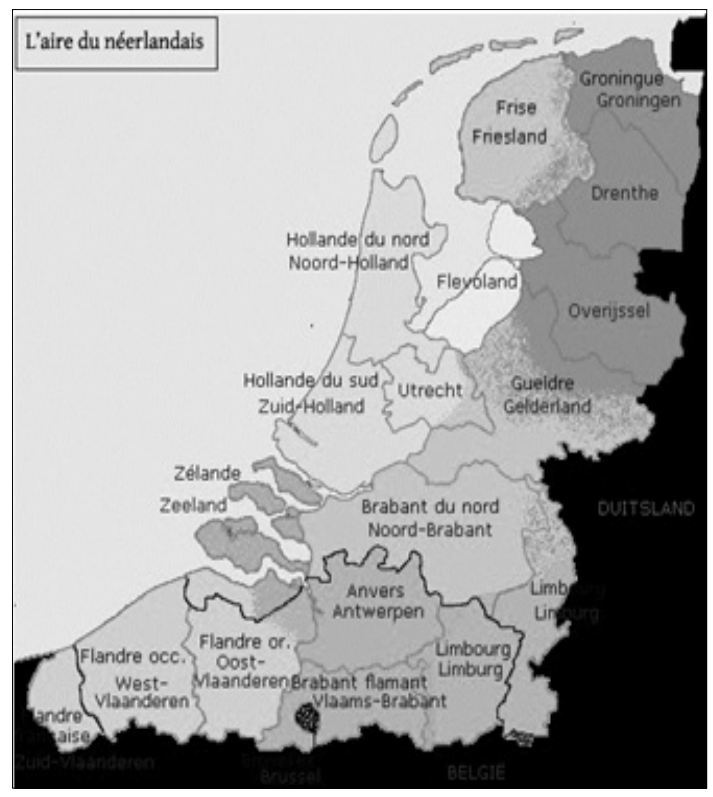

\section{Le bas-allemand}

Historiquement, le néerlandais s'est formé à l'extrémité occidentale du vaste domaine germanique de la mer du Nord ou ingvaeonique ${ }^{3}$ qui

3. Les Ingaevones étaient une proto-tribu qui vivait le long de la côte de la mer du Nord et constituait un groupe culturel germanique occidental. 


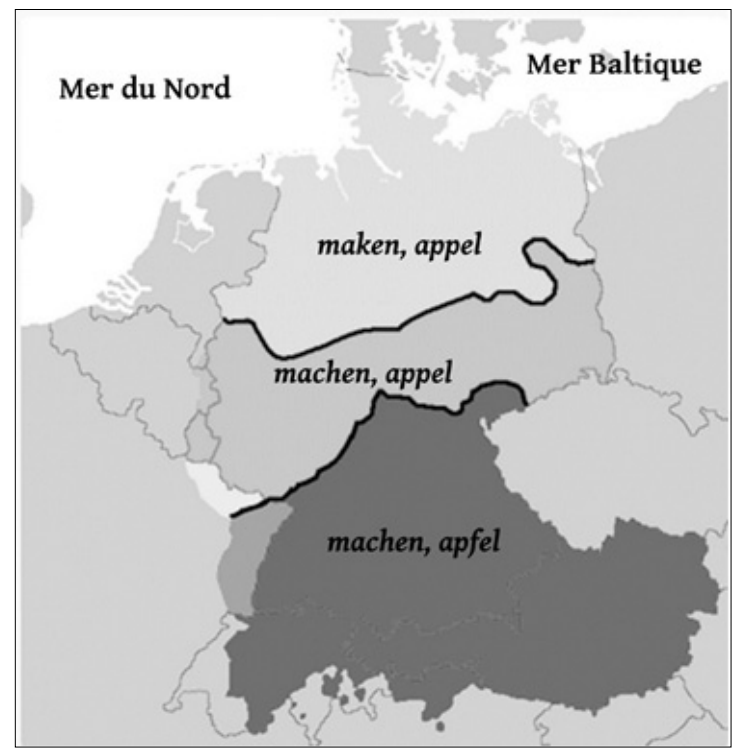

L'aire du bas-allemand

regroupe l'anglais, le frison, le néerlandais et le bas-allemand actuels. Le néerlandais appartient précisément à l'aire du bas-allemand, qui s'étend de Dunkerque aux confins de la Pologne ${ }^{4}$. Au Moyen Âge on ne le distinguait pas de l'allemand, avec lequel il constituait la lingua theodisca, du germanique peudisk "peuple ${ }^{5}$ par opposition au latin, langue des clercs et des lettrés. C'est de ce mot que dérivent les termes Deutsch, dietsch, duutsch et le français thiois qui, aujourd'hui encore, désigne le francique lorrain. En anglais, le néerlandais est appelé dutch.

Aucune isoglosse ne sépare les territoires de l'allemand et du néerlandais actuels. En revanche, le continuum septentrional du bas-allemand se distingue en bloc des aires du moyen et du hautallemand.

Des isoglosses permettent de partager le domaine germanique occidental en trois zones à partir de critères phonétiques portant sur ce que les philologues nomment la deuxième mutation consonantique ${ }^{6}$.

4. Et jusqu'à Königsberg avant l'expulsion des Allemands d'Europe de l'Est à la fin de la Seconde Guerre mondiale.

5. En vieil-anglais pēodisc, et en vieux-haut-allemand diutisc. Le mot est lointainement apparenté au breton tud de même sens.

6. La première mutation consonantique est celle qui fait que, selon la doctrine indoeuropéaniste, le germanique aurait transformé les consonnes de l'Indo-Européen, 
On constate ainsi les correspondances suivantes : maken / machen "faire», appel IApfel "pomme», schip / Schiff "navire», toll / Zoll «douane» 7 .

Au nord, la ligne Benrath (dite aussi ligne machen / maken) va de Eupen (Belgique) jusqu'au Rhin (vers Benrath, près de Düsseldorf), puis se dirige vers Francfort-sur-l'Oder en contournant Berlin. Elle sépare le bas-allemand au nord du moyen-allemand (Mitteldeutsch), une zone de transition, où seule une partie des consonnes est affectée par la mutation. La ligne est bien entendu approximative : un peu au nord de la ligne Benrath, une autre ligne dite de Uerdingen sépare l'aire septentrionale où «je» se dit $i k$, comme en néerlandais, de celle où il se dit $i c h$ comme en haut-allemand. On observe d'autres différences, par exemple le passage de $d$ - à $t$ - en haut-allemand (duur vs teuer "cher») et des variations diverses dans le système vocalique. Au sud, la ligne dite de Speyer sépare le moyen-allemand du haut-allemand au sud où toutes ces mutations se produisent. Il semble donc que les changements se soient produits au sud pour s'étendre ensuite vers le nord, en vagues d'inégale amplitude et à des dates éminemment variables selon les auteurs.

On divise traditionnellement l'aire du bas-allemand en "dialectes» à partir de critères plus historiques ou géographiques que linguistiques. D'est en ouest on distingue le bas allemand oriental (Nord-Est de l'Allemagne), le bas allemand occidental, ou bas-saxon qui couvre une petite aire au Nord-Est des Pays-Bas et enfin le bas francique de Flandre et de Flandre Zélandaise correspondant à l'ancien royaume des Francs, d'où procède le néerlandais actuel. En fait, on a affaire à une juxtaposition de badumes comme on en trouvait partout en Europe quel que fût le domaine linguistique. Ces régions étant longtemps demeurées des entités politiques différentes, leurs badumes se sont conservés plus tardivement qu'en France.

ce qui expliquerait par exemple qu'au latin piscis "poisson» correspond l'allemand Fisch, l'anglais fish et le néerlandais vis.

7. Le suisse allemand va au bout de la mutation à l'initiale : l'allemand a pf- (Pfeife "pipe») et ts (Zwei "deux»), mais Kind "enfant», tandis qu'en Suisse on entend ( $k$ ) chind pour ce dernier mot. 
Les badumes néerlandais sont aujourd'hui pratiqués de façon très inégale aux Pays-Bas et en Belgique. Dans les premiers, pour des raisons historiques que nous exposerons plus loin, ils sont en net recul devant la langue générale ${ }^{8}$, tandis qu'en Flandre, bien que concurrencés par la tussentaal "langue intermédiaire ${ }^{9}$ ", les dialectes des marges comme le West-Vlaams "flamand occidental» en usage dans la province belge de Flandre occidentale, en Flandre française et à l'extrême ouest de la province néerlandaise de Zélande constituent des particularismes encore si vivaces de nos jours que les propos de leurs locuteurs sont sous-titrés en néerlandais "civilisé» dans les émissions des télévisions flamandes et néerlandaises ${ }^{10}$.

Comme dans tous ces "dialectes", chaque badume a son propre nom : ainsi, à Zwevegem près de Courtrai on parle le zwevegems. Une variété de flamand occidental survit aussi chez des personnes âgées dans le Westhoek «coin de l'ouest», petite région du Nord de la France proche de Dunkerque. Le brabançon est encore très parlé, particulièrement à Anvers. Les badumes ne connaissent pas de frontières : certains parlers comme le limbourgeois sont à cheval entre la Belgique, les Pays-Bas et l'Allemagne : le parler de Bentheim (Allemagne) est plus proche du néerlandais officiel que celui de Maastricht (Pays-Bas) ou de Hasselt (Belgique). En-dehors du domaine néerlandais, on trouve d'autres badumes comme le bas-saxon sur les confins orientaux des PaysBas historiques (actuelles provinces de Drenthe, de Overijssel et de Groningue) et le frison en Frise.

\section{L'apparition des standards}

On observe à certaines époques la naissance et le développement de structures linguistiques stables correspondant au développement de ce que nous nommons des «institutions coutumières» dont les

8. Ce qui n'est sans doute pas si sûr quand on lit sur un blog Ik kom uit het land waar je doorheen rijdt in 3 uurtjes, met een ander dialect elke 10 minuutjes "Je viens du pays que tu traverses en 3 heures avec un dialecte différent toutes les 10 minutes».

9. Voir l'article de Gudrun Ledegen sur la tussentaal dans ce volume.

10. Depuis peu certaines séries télévisées en flamand occidental ne sont plus soustitrées, ce qui indiquerait qu'ils seraient compréhensibles par les auditeurs d'autres régions. En revanche, la télévision néerlandaise sous-titre parfois les propos d'un Flamand cultivé s'exprimant pourtant en une langue normée. 
règles sont justifiées par la tradition et le sens commun. En usage dans des domaines sociaux divers - religion, commerce, justice - elles fonctionnent à l'échelle d'une région plus vaste que les badumes. Nous les nommons standards, faute de terme plus adapté et au risque de confusion avec ce que nous appelons norme (voir plus bas). Dépassant le cadre purement local et les besoins quotidiens, le standard gomme partiellement la variation des badumes dans le but de créer un espace de communication qui corresponde à cette institution, à l'usage de ceux qui ont besoin de l'écriture dans une société encore massivement analphabète. Apanage d'une minorité, sa relative régularité permet de le transporter dans toute une principauté féodale, dans toute une zone économique, quelle que soit la diversité des badumes qu'il recouvre.

Au sein de la Ligue hanséatique, association de villes marchandes de la mer du Nord qui, de 1200 à 1550, a commercé jusqu'en Russie et en Italie s'est élaboré un standard écrit remplaçant le latin pour la rédaction d'une multitude de documents, contrats et livres. Fondé à l'origine sur le bas-allemand de Lübeck, il a servi de langue commerciale, juridique, diplomatique et littéraire tout au long des côtes de la mer du Nord et au-delà : la Bible de Lübeck en bas-allemand a été publiée en 15331534. Mais ce standard s'est éteint avec la fin de la Ligue, et il n'a jamais été la langue d'un État : la disparition de l'institution entraîne celle de son standard. Seuls subsistent jusqu'à nos jours des badumes plus ou moins en voie d'abandon ${ }^{11}$.

\section{Les standards du Sud dominent}

Selon la tradition allemande du XIX ${ }^{\mathrm{e}}$ siècle qui assimile les langues à des êtres vivants, les philologues découpent l'histoire des langues comme s'il s'agissait de tranches de vie, de passages obligés. Ils distinguent ainsi le vieux néerlandais (800-1150), le moyen-néerlandais (1150-1500), le néerlandais prémoderne (1500-1700), le néerlandais moderne (17001900) et enfin le néerlandais contemporain (1900 à nos jours). De notre point de vue, tous les avatars de ce qu'on classe sous le terme générique de "langue» dépendent, non pas d'une évolution interne, mais des besoins des institutions humaines qui suscitent leur création.

11. En revanche, le néerlandais normé de Hollande jouera aux XVII ${ }^{\mathrm{e}}$ et XVIII ${ }^{\mathrm{e}}$ siècles le rôle de langue diplomatique dans les ports de la Baltique. 
Leur existence et leurs transformations sont soumises aux fluctuations de l'histoire.

Le texte traditionnellement ${ }^{12}$ tenu pour être la première attestation du néerlandais écrit est la traduction littérale d'une phrase latine par un moine flamand séjournant dans le monastère de Rochester (Kent) en Angleterre vers 1100 : Hebban olla vogala nestas hagunnan hinase hic anda thu, wat unbidan we nu? "Tous les oiseaux ont commencé à faire leurs nids, sauf moi et toi, qu'attendons-nous ?». Ce qui rappelle le fameux texte breton d'Ivonet Omnes de 1350 (an guen heguen am laouenas...). Il s'agissait probablement pour ce scribe d'essayer une nouvelle plume d'oie (probatio pennae) en écrivant ce qui lui passait par la tête ... On ne peut cependant pas parler de standard ${ }^{13}$ dans cet exemple : à ces époques lointaines, le latin était d'usage universel dans l'Europe chrétienne. Si un tel texte est précieux d'un point de vue symbolique, peut-on vraiment affirmer qu'il s'agit d'un ancêtre du néerlandais?

Au moyen âge, les villes flamandes comme Bruges, où se trouvait la chancellerie du comte, étaient prospères, et c'est en leur sein que vont se développer jusqu'en 1300 les deux-tiers des standards néerlandais dans les domaines politique, administratif et commercial. Même si on les caractérise comme flamands, brabançons ou hollandais, il s'agit en fait de variétés écrites diverses bien que mutuellement compréhensibles. On peut cependant considérer que c'est à ce moment que commence l'histoire de la future langue néerlandaise. Le plus ancien texte à nous être parvenu intégralement est De statuten van de Gentse leprozerie (1236) "Les Statuts de la léproserie de Gand" par lesquels l'évêque de Tournai imposait au personnel infirmier de l'établissement le respect des vœux de chasteté, de pauvreté et d'obéissance. Ces standards, rassemblés sous le nom générique de diets, étaient en rapport de colinguisme avec le français ${ }^{14}$. Depuis le traité de Verdun (843) partageant l'empire de Charlemagne entre ses fils, une des principales frontières politiques de

12. D’autres écrits de cette époque ont été trouvés depuis la date de la découverte de celui-ci en 1932, qui garde cependant sa réputation de premier texte en vieuxnéerlandais.

13. Et certains pensent même qu'il pourrait s'agir de vieil-anglais !

14. Renée Balibar, Le colinguisme, Paris, PUF, coll. "Que sais-je ?», n² 2796, 1993. 
l'Europe occidentale traversait l'actuelle Belgique du sud au nord en suivant le cours de l'Escaut. Ce qui signifie que le comté de Flandre relevait du Royaume de France, tandis que ceux qui deviendraient les Wallons, de langue romane, se trouvaient rattachés à une entité de langue germanique. Le sud de la Flandre de langue française s'appelait la Flandre gallicante par opposition à la Flandre flamingante ${ }^{15}$. L'aristocratie flamande était en étroit contact avec la noblesse picarde ou artésienne, et son standard s'est très tôt appelé flamand en français, nom qui s'est appliqué par métonymie aux parlers des autres régions comme le Brabant ou le Limbourg, voire à la langue néerlandaise en général ${ }^{16}$.

Des échanges actifs se sont poursuivis, comme en témoignent les nombreux guides de conversation français-flamand, dont au $\mathrm{XIV}^{\mathrm{e}}$ siècle le Livre des mestiers, manuel pratique «en rommans et flamenc", composé par un maître d'école inconnu et publié à Bruges au $\mathrm{XIV}^{\mathrm{e}}$ siècle à destination des étrangers désireux d'apprendre le flamand. C'est l'un de ces colloques, paru pour la première fois à Anvers en 1530, qui a été adapté en breton et réédité jusqu'au début du XXe siècle, servant de manuel de français aux bretonnants ${ }^{17}$. On sait la porosité des modèles et des thèmes qui circulaient dans l'Europe médiévale en latin et en standards locaux : le succès du Roman de Renard (Van den Vos Reynaerde), initialement composé en Flandre en latin, a provoqué le remplacement en français du mot goupil par renard, tandis que le flamand subissait une forte influence française, à l'instar de l'anglais et du breton.

\section{Les Dix-Sept Provinces}

Cette influence française se poursuivit au $\mathrm{XV}^{\mathrm{e}}$ siècle lorsque les principautés couvrant les territoires de la Belgique et des Pays-Bas actuels furent réunies sous l'autorité du Grand Duc de Bourgogne puis des Habsbourg d'Espagne. À Bruxelles, la langue française commençait à être utilisée à côté du brabançon et d'un peu d'espagnol. Quand

15. En Bretagne on opposait de même la Brittania gallicana à la Brittania britonizans. 16. De la même façon la peinture flamande est l'œuvre de peintres originaires de tous les anciens Pays-Bas.

17. Adolphe LE GOAZIOU, La longue vie de deux colloques françois et breton (16261915) : essai de bibliographie bretonne, Quimper, Le Goaziou, 1950. 
le port de Bruges perdit son accès à la mer à cause de l'ensablement du Zwin, c'est le Brabant, situé sur la route commerciale LondresCologne, qui va dominer politiquement et économiquement à partir de 1400 - à partir de villes comme Anvers, Malines, Louvain. Les comtes de Bourgogne, résidèrent à Bruxelles dès 1430 et leur administration était bilingue. Une production écrite se développa en un standard brabançon appelé généralement nederduits. L'unification politique amorcée par les Bourguignons se poursuivit sous Charles Quint, qui réunit au $\mathrm{XV}^{\mathrm{e}}$ siècle les Dix-Sept Provinces, rassemblant les territoires actuels des Pays-Bas, de la Belgique, du Luxembourg, du Nord-Pas-deCalais et de quelques territoires allemands.

Une rupture décisive : la séparation entre Nord et Sud

Le Traité de Westphalie de 1648 mit fin à la guerre de QuatreVingts Ans, également appelée révolte des Gueux, un soulèvement armé mené de 1568 à 1648. Il aboutit à l'indépendance des Provinces-Unies - Pays-Bas actuels : Hollande, Zélande, Utrecht, Gueldre, Overijssel, Frise, Groningue (avec Drenthe). Les dix provinces du sud, parfois appelées Pays-Bas du sud, - Flandre, Artois, Malines, Anvers, Hainaut, Namur, Brabant, Limbourg, Luxembourg, auxquels est joint Cambrai - demeuraient aux mains des Espagnols catholiques. La domination étrangère n'allait se terminer qu'en 1830, avec la création de la Belgique.

Cette coupure entre les territoires actuels de la Belgique néerlandophone et les Pays-Bas a marqué l'histoire du néerlandais de façon indélébile. Le Sud avait subi une forte influence du protestantisme : c'est à Steenvoorde, dans le Westhoek (maintenant français), qu'eut lieu le premier saccage d'un lieu de culte, le couvent de Saint-Laurent, par des protestants iconoclastes. Cette révolte s'est par la suite étendue à l'ensemble des Pays-Bas du Sud, puis à Anvers, devenue république d'Anvers ou république calviniste entre 1577 et 1585, place forte du protestantisme. Le siège d'Anvers s'acheva en 1585 par une victoire espagnole. La ville perdit alors son statut de plaque tournante de l'économie européenne au profit d'Amsterdam en raison du blocus appliqué par la flotte hollandaise sur l'Escaut. La ville fut ruinée, et la moitié de ses habitants participa à l'exode massif au XVII siècle de quelque 100000 à 150000 réfugiés vers le Nord, en majorité des 
protestants flamands et brabançons. La population de la Hollande et de la Zélande s'accrut ainsi de 15 à $20 \%$.

Dès lors, l'opposition entre Flandre et Pays-Bas ne va faire que se creuser. D'abord pour des raisons religieuses évidentes : alors que le sud demeurait ou redevenait catholique, c'est le calvinisme qui va régner au nord, favorisant les professions commerciales et industrielles, tandis que le Sud de la zone néerlandophone restait essentiellement rural, fortement marqué par le catholicisme et attaché aux valeurs traditionnelles.

\section{La norme : naissance du néerlandais}

Le troisième et ultime étage de notre construction concerne les «institutions de droit» dont les règles sont toujours écrites et justifiées par la raison universelle, en usage dans le cadre d'un État national, dans tous les domaines de la vie sociale, établissant une égalité de droit entre tous les citoyens : l'archétype en est évidemment le français. Nous appelons l'idiome qui leur correspond la norme, qui s'impose à tous et appartient à chacun sans constituer en tant que telle la parole de personne. Chaque norme fait l'objet de discours renvoyant à des normes évaluatives et fictives, mais aussi à la rédaction de normes prescriptives $^{18}$.

Les Provinces Unies du XVII ${ }^{e}$ siècle étaient une république calviniste forte, prospère et conquérante, une puissance maritime et commerciale de premier plan, relayant dans ce rôle l'Espagne du $\mathrm{XVI}^{e}$ siècle avant d'être supplantée par l'Angleterre du XVIII ${ }^{e}$ siècle. Leur commerce maritime couvrait une grande partie du monde grâce à leurs Compagnies maritimes, véritables multinationales ${ }^{19}$ avant l'heure, qui nécessitaient la mise au point d'une norme compréhensible par tous les participants et basée sur la variété hollandaise détentrice du pouvoir. Cette norme, utilisée par les compagnies maritimes, était

18. Constatons que le mot langue, quoique recouvrant des réalités très différentes, s'applique aussi bien au badume local, au standard régional qu'à la langue nationale, d'où l'impossibilité de lui donner une définition scientifique unique.

19. Compagnie néerlandaise des Indes orientales (Vereenigde Oostindische Compagnie, 1602-1799) et Compagnie néerlandaise des Indes occidentales (Geoctroyeerde Westindische Compagnie, 1621-1792). 
également cultivée dans les universités, dont celles de Leyde et de Groningue, tandis que des écrivains et savants humanistes tels Joost van den Vondel (1587-1679), Pieter Corneliszoon Hooft (1581-1647) et Gerbrand Adriaenszoon Bredero (1585-1618) composaient les premiers "classiques» de la littérature néerlandaise.

On lit couramment que les immigrés du Sud des Pays-Bas auraient eu un rôle majeur dans le développement de la norme au XVII siècle. Il s'agissait en effet pour une bonne part de riches marchands, d'ouvriers spécialisés, mais aussi d'intellectuels exilés tant par nécessité que par conviction, dont un grand nombre de maîtres d'école, d'imprimeurs, de libraires, mais aussi de pasteurs protestants. La traduction officielle de la Bible en néerlandais, la célèbre Statenbijbel «Bible des États Généraux» de 1637, commandée par le Parlement au Synode de Dordrecht en 1618, aurait été fortement marquée par des traducteurs venus du Sud. Pour la linguiste néerlandaise Nicoline van der Sijs ${ }^{20}$, on ne trouve dans la norme actuelle pratiquement aucune influence des parlers du sud remontant à cette époque. En revanche, elle considère que de nombreux traducteurs bibliques venus de l'Est ou d'Allemagne auraient marqué la norme, et que les grammairiens du temps faisaient souvent référence à l'allemand et non aux dialectes du sud. La traduction néerlandaise de la bible de Luther parue en 1526 à Anvers, soit un siècle avant la Statenbijbel, aurait selon la linguiste fortement influencé la langue normée. Pour elle, le mythe de la prédominance des dialectes du Sud aurait été un argument des Flamands intégrationnistes de la fin du XIX ${ }^{e}$ siècle justifiant l'adoption par la Belgique de la norme déjà fonctionnelle des Pays-Bas, État encore souvent considéré comme ennemi, tout en réduisant le poids de la langue allemande considérée par beaucoup comme trop dominante et en s'éloignant autant que possible de l'influence française. La discussion est ouverte...

20. Nicoline VAN DER SIJS, Taal als mensenwerk, de geschiedenis van het ontstaan van het $A B N$ [La langue en tant que création humaine, l'histoire des origines de l'ABN], Den Haag, SdU Uitgevers, 2004. Dans cet ouvrage l'auteure minimise l'influence des Flamands émigrés et de la Statenbijbel dans la création de la norme et souligne la forte influence de la langue allemande. 


\section{Les Pays-Bas méridionaux, future Belgique}

En 1612 les jésuites, soucieux comme partout - comme le père Maunoir en Bretagne - de convertir le peuple, divisèrent la province ecclésiastique de Belgique en deux parties presque égales sur la base des langues, sans tenir compte des divisions administratives ni des divisions politiques préexistantes, préfigurant ainsi la Belgique actuelle ${ }^{21}$. Leur influence sur la recatholicisation fut importante : ils créèrent un nombre impressionnant d'écoles, très ouvertes dans leur fonctionnement. Les capucins suivirent et augmentèrent encore le poids de l'Église sur le peuple. Si les dirigeants parlaient les deux langues, c'est dans la pratique le français qui servait à la communication administrative. Le peuple de Bruxelles utilisait encore majoritairement le brabançon, mais la ville se francisait, d'abord en raison du prestige européen du français, mais aussi par l'arrivée de protestants français. En 1792, quand la France envahit les Pays-Bas autrichiens, chacun utilisait toujours son badume, qu'il soit germanique ou roman, et on comptait beaucoup d'illettrés.

\section{Période révolutionnaire (1792-1814)}

La France occupa les Pays-Bas autrichiens (soit les Pays-Bas méridionaux, comprenant la Flandre actuelle) de 1792 jusqu'à la chute de l'Empire en 1814. Pendant cette période le français régnait seul dans la vie publique. La francisation de l'élite bourgeoise fut très rapide, $y$ compris au sein de la classe moyenne néerlandophone, qui en vient désormais à considérer le français comme sa langue. Les standards régionaux furent abandonnés, seuls subsistèrent les badumes. Ces années bouleversèrent l'organisation administrative et législative préexistante, marquant la fin des privilèges. Aucun retour en arrière n'eut lieu par la suite. C'est en 1796 que fut créé un registre d'état civil, avec l'obligation pour chacun d'adopter un nom de famille.

21. Il n'existait pas et n'avait jamais existé de découpage administratif flamand, bien que le mot «flamand" ait pu désigner par synecdoque l'ensemble des Pays-Bas espagnols. On n'a jamais désigné les parlers du Brabant ou du Limbourg sous le nom de flamand. Il n'y a jamais eu non plus de regroupement des Picards, Wallons et Lorrains, qui n'ont tous été rassemblés sous le nom de Wallons au sein d'une même entité que dans la Belgique du XIX siècle pour aboutir à une Belgique duale (à laquelle s'ajoutent les petits territoires germanophones). 


\section{La République batave (1795-1806)}

Après une révolution ratée au sein des Provinces Unies en 1787, la France envahit le pays et créa une république "une et indivisible» de brève durée, mais aux conséquences très importantes : le pays fut centralisé à la française et réorganisé administrativement. Comme dans le Sud, on mit en place en 1811 un registre d'état civil introduisant l'usage obligatoire de noms de famille. Les Compagnies furent dissoutes, et le pays perdit des colonies au profit de l'Angleterre. Ceci explique pourquoi la langue néerlandaise ne s'est pas répandue ailleurs qu'au Surinam, et n'ait gardé d'usage administratif que dans les Antilles néerlandaises. À cette époque aussi l'unification de la langue et de son orthographe fut accentuée. On créa pour cela dès 1797 une chaire de langue néerlandaise et d'éloquence à Leyde.

Une période charnière révélatrice : le Royaume Uni des Pays Bas (1815-1830), état tampon

À la chute de l'Empire, le congrès de Vienne créa en 1815 un État tampon entre les grandes puissances européennes, le Royaume Uni des Pays-Bas, rassemblant en gros les actuels Pays-Bas - république protestante prospère et unifiée - et la future Belgique - vague confédération de principautés catholiques. Deux cents ans après la rupture entre les deux entités et leur développement séparé, le Sud et le Nord furent ainsi de nouveau réunis par la force. Alors que le Nord avait désormais une norme établie, le Sud restait partagé entre un peuple ne parlant essentiellement que des badumes germaniques ou romans et une bourgeoisie industrielle entièrement francophone commune à la Wallonie et à la Flandre issue de l'époque française. Aussi, quand le roi Guillaume Ier d'Orange voulut en 1829 imposer le néerlandais comme langue de la justice et de l'administration dans les provinces néerlandophones afin de lutter contre la propagation des idées révolutionnaires, il provoqua un fort mouvement de révolte parmi la bourgeoisie : l'exclusion du français de l'administration lui était intolérable, et elle ne pouvait admettre qu'à Bruxelles, devenant une ville néerlandophone, on n'étudierait même pas le français. Quant au peuple, qui n'avait plus depuis des siècles de liens culturels avec les PaysBas du Nord, il était réfractaire à ce "hollandais» - comme on appelle couramment le néerlandais des Pays-Bas - qu'on voulait lui imposer, 
tandis que les ecclésiastiques s'en méfiaient en tant que véhicule du calvinisme.

\section{La Belgique : le règne du français}

En 1830, quand les provinces du Sud obtinrent leur indépendance, la Wallonie dominait économiquement et linguistiquement, tandis que la partie néerlandophone restait rurale et très pauvre. Beaucoup de Flamands émigraient et adoptaient le français, comme les Irlandais le faisaient avec l'anglais. La Constitution de 1830 stipulait dans son article 23 que "l'emploi des langues est facultatif en Belgique, il ne peut être réglé que par la loi et seulement pour les actes de l'autorité publique et pour les affaires judiciaires ». Mais dans les faits c'est le français, langue de la classe dominante et des élites européennes, qui fut choisi comme seule langue officielle à Bruxelles, mais aussi en Flandre : seule la version française des textes de loi était officiellement valable.

\section{Une difficile reconnaissance}

Il fallut attendre 1850 pour que l'enseignement des deuxièmes langues du pays (flamand ou allemand) soit rendu obligatoire là où elles étaient en usage à côté du français. Le bilinguisme fut officiellement déclaré en 1873. En 1878 la Gelijkheidswet «loi d'égalité» réglementa l'usage des langues en matière administrative en définissant trois territoires linguistiques au sein de la Belgique, mais ce n'est qu'en 1898 que les deux langues furent mises sur le même pied du point de vue juridique. Bruxelles demeura officiellement francophone jusqu'en 1883 jusqu'à ce qu'on autorisât le "flamand" dans l'enseignement primaire en 1889 et dans les tribunaux.

L'enseignement primaire était bilingue, mais le supérieur uniquement en français. La démocratisation de l'enseignement au $\mathrm{XIX}^{\mathrm{e}}$ siècle multiplia cependant le nombre d'enseignants et d'étudiants sensibles à la cause flamande, - et, ultérieurement, le nombre de «flamingants» parmi les professions libérales. À côté des capitalistes francophones apparaissaient des chefs d'entreprises flamands. Pendant l'entre-deux guerres, la cause nationaliste ne s'exprimait plus seulement sous la forme de la simple revendication linguistique mais s'y ajoutaient des arguments socio-économiques. Un événement important fut la lutte victorieuse de l'université d'État de Gand pour sa néerlandisation en 
1930 sous l'égide du Frontpartij «Parti du Front» issu du Frontbeweging "Mouvement du Front» créé par des soldats qui se plaignaient d'avoir été commandés uniquement par des francophones sur le front de l'Yser pendant la Première Guerre mondiale. La lutte fut très rude, car son issue constituait une défaite pour la bourgeoisie francophone.

Une loi de 1931 créa trois régions linguistiques : une région de langue néerlandaise, une région de langue française et une région bilingue dans l'agglomération bruxelloise. Pour cela on délimita une "frontière linguistique» dont le tracé pouvait évoluer en fonction de recensements linguistiques décennaux. Pour qu'une région soit déclarée bilingue, il fallait que $30 \%$ de la population déclare ne pas parler la langue majoritaire de sa région. Comme les recensements décennaux marquaient des évolutions dans la frontière linguistique au profit du français, une loi de 1962 décréta le "clichage» définitif de la frontière linguistique, ce qui signifie qu'elle ne pourrait plus être modifiée, en accordant des "facilités» aux communes bilingues de part et d'autre. La Flandre devient ainsi officiellement une région unilingue néerlandophone et la Wallonie unilingue francophone.

Cela entraîna nombre de contestations : la plus importante fut le rattachement de la commune de Fourons (4000 habitants) à la province de Limbourg, qui constitua l'un des plus importants points de cristallisation du différend entre les communautés, provoquant la démission d'un ministre et même d'un gouvernement. Cette commune, dont la population parlait un badume platt-dütsch rhéno-mosan, très proche de celui d'Aix-la-Chapelle et qui est utilisé dans les communes dites germanophones de Belgique, dépendait auparavant de Liège. Les enfants étudiaient en néerlandais en primaire, en français ensuite en secondaire, et parlaient le badume à la maison. Désormais, la commune s'est scindée entre partisans du néerlandais et partisans du français, avec pour résultat la disparition du badume!

En 1960 la radio-télévision fut scindée en deux instituts, un flamand et un wallon; en 1970, on accorda l'autonomie culturelle aux Flamands et aux Wallons; en 1980, on répartit la santé et l'aide sociale entre deux communautés : c'était le début d'une séparation qui ne fit que s'accentuer avec la fédéralisation de l'État qui survint en 1993. 


\section{Le choix du néerlandais}

Les Belges et les Hollandais étaient différents voire opposés tant par la religion que par la culture - comme l'a démontré la malheureuse expérience du Royaume uni. En 1830, il n'existait pas de norme flamande. La langue écrite conservait de nombreux traits archaïques fortement variables selon le parler natif des auteurs, comprenant des termes locaux et de nombreux calques du français. En conséquence, un courant particulariste, centré sur les régions agricoles fortement catholiques où se parlait le flamand occidental, dont le prêtre et poète Guido Gezelle (1830-1899) était une figure marquante, tentait d'établir une norme flamande qui permettrait de s'opposer à la néerlandisation de la Flandre, dont ils craignaient qu'elle risquât de provoquer un retour $\mathrm{du}$ protestantisme ${ }^{22}$. Dans cette période initiale, la revendication, menée essentiellement par le petit clergé et la petite bourgeoisie, était purement de nature linguistique et ne recherchait pas l'indépendance politique. Une de ses figures de proue était Henri Conscience, l'auteur du célèbre roman historique De Leeuw van Vlaenderen "le Lion des Flandres» paru en 1838. Son père trouva si inconvenant qu'il écrive en «flamand» et non en français qu'il le mit dehors! Le roman a connu plusieurs révisions par la suite, car sa langue et son orthographe étaient défectueuses.

Par ailleurs, les intellectuels qui avaient fait leurs études sous le régime hollandais et connaissaient donc parfaitement le néerlandais normé ont été frustrés quand ils sont devenus citoyens d'un État belge dans lequel le français régnait dans la vie publique. Opposés de fait aux "fransquillons", la grande bourgeoisie francophone de Flandre, ils sont à la base de la naissance du mouvement flamand, mouvement d'émancipation sociale qui visait au début à mettre un frein à la francisation et à rétablir le néerlandais dans l'administration, la justice et l'enseignement. Ce sont eux qui gagnèrent ce combat : à leurs yeux, l'ancien flamand était voué à disparaître. Comme l'écrit en 1998 Astrid von Busekist :

"La Flandre abritait un ensemble disparate de dialectes, appartenant à la famille linguistique du thiois, mais dont les différents patois étaient

22. Cette entreprise née en Flandre occidentale ne concernait pas les provinces de Flandre orientale, du Brabant, d'Anvers et du Limbourg. 
circonscrits géographiquement, souvent dans les limites du hameau ou de la commune ${ }^{23}$. Seuls les locuteurs néerlandophones éclairés, appartenant à la classe privilégiée, et maîtrisant par conséquent le français, avaient une conscience de la langue et pouvaient en faire un objet de réflexion et de comparaison. Ces locuteurs se divisaient en deux groupes, dont l'un souhaitait élaborer un flamand standardisé, respectueux de la tradition culturelle propre aux provinces flamandes, mais néanmoins dans un rapport de proximité orthographique et grammaticale avec le néerlandais des Pays-Bas, et l'autre admettait la supériorité du français comme langue véhiculaire à l'intérieur de l'État ${ }^{24}$ ".

Porté à ses débuts par une bourgeoisie urbaine libérale, le mouvement va adopter une posture droitière et catholique après l'instauration du suffrage universel en 1919 en remplacement du suffrage censitaire qui favorisait les francophones, introduisant à la vie politique la majorité numérique dont les masses flamandes rurales disposaient. C'est le moment où les Belges néerlandophones se sont mués en peuple flamand. En miroir se développera un peuple wallon libéral puis socialiste.

Jan Frans Willems (1793-1846), considéré comme le père du Mouvement flamand, est l'une des figures marquantes de ceux qu'on a appelés les intégrationnistes. Il s'était déjà opposé dans un pamphlet de 1824 Over de Hollandsche en Vlaemsche schryfwyzen van het Nederduitsch "Sur les orthographes hollandaise et flamande du bas-allemand [néerlandais]» aux particularistes, partisans d'une orthographe propre à la Belgique, le système de Jan Des Roches. Le Mouvement flamand se mit en contact avec les Pays-Bas afin de renforcer sa position et prit l'initiative d'organiser les Congrès linguistiques et littéraires néerlandais ${ }^{25}$, dont le premier eut lieu à Gand en 1849. Des écrivains et des universitaires des deux pays coopérèrent, et mirent en place plusieurs projets, dont la mise en chantier du Woordenboek der Nederlandsche Taal «Dictionnaire de la langue néerlandaise» : terminé en 1998, il serait, dit-on, le plus long dictionnaire au monde. L'orthographe uniformisée dite de De Vries-Te Winkel du nom de leurs créateurs fut appliquée en 1864 en Belgique et en 1883 aux Pays-Bas. En 1927, une norme commune aux deux pays fut

23. Ce qui correspond exactement à notre définition des badumes.

24. Astrid VON BUSEKIST, La Belgique. Politique des langues et construction de l'État de 1780 à nos jours, Bruxelles, De Boeck \& Larcier, 1998, p. 47.

25. Nederlandsche Taal-en Letterkundige Congressen. 
adoptée officieusement sous le nom de Algemeen Beschaafd Nederlands (ABN), qui signifie littéralement «Néerlandais général cultivé». L’adjectif beschaafd "cultivé» fut abandonné vers 1970 en raison de sa résonance élitiste, et on parle désormais de Algemeen Nederlands "néerlandais général» ou de Standaardnederlands "néerlandais standard». Tous ces efforts aboutirent, en 1946, à la signature d'un traité culturel entre les Pays-Bas et la Belgique, par lequel la Belgique adoptait officiellement la norme néerlandaise des Pays-Bas, avec une orthographe commune. On ne parle plus désormais de Vlaams «flamand» mais de nederlands «néerlandais». En 1995 fut créé le Vlaamse Culturele Raad "Conseil Culturel Flamand», organisme indépendant permettant la signature de traités avec d'autres pays. Dès son entrée en vigueur le Traité culturel belgo-néerlandais devint le Cultureel Verdrag tussen Nederland en Vlaanderen "Traité culturel entre les Pays-Bas et la Flandre».

\section{L'Union de la langue néerlandaise (1980)}

Ce n'est qu'en 1980 que fut fondée entre la Flandre et les PaysBas la Nederlandse Taalunie "Union de la langue néerlandaise», pour unifier les questions relatives à la langue néerlandaise, y compris son enseignement à l'étranger (par exemple en France dans le Nord-Pas de Calais). Le Suriname a rejoint cette Union en 2005, et les îles Aruba, Bonaire et Curaçao également dans une certaine mesure (bien que dans ces îles la langue la plus parlée soit le papiamento, un créole lusoespagnol). Un important projet de l'Union a été la modification de l'orthographe néerlandaise en 1995, après l'importante réforme de 1946. Elle publie la Woordenlijst Nederlandse Taal "Glossaire des mots de la langue néerlandaise» familièrement appelée Het Groene Boekje "le petit livre vert», dont la première version est parue en 1865 sous le titre Woordenlijst voor de spelling der Nederlandsche taal «Glossaire de l'orthographe de la langue néerlandaise» par De Vries en Te Winkel. La dernière version en est parue en 2005, mais en 2008 la Taalunie a annoncé qu'il n'y aurait plus de nouvelle révision orthographique en 2015, qu'on ne ferait qu'y ajouter les mots nouveaux. À côté de cette liste, la référence officieuse mais très suivie est le Groot woordenboek van de Nederlandse taal "grand dictionnaire de la langue néerlandaise» de Van Dale, dont la quinzième édition est parue en 2015. 


\section{La situation présente en Belgique}

Les rapports de force entre Flandre et Wallonie se sont inversés depuis les années 1960. Parallèlement au déclin économique de la Wallonie, la Flandre, au climat social plus paisible, a développé un réseau d'entreprises petites et moyennes, souples et modernes, et elle a accueilli un grand nombre d'entreprises étrangères.

Pour s'opposer aux exigences flamandes, la francophonie bruxelloise a fait son apparition sur la scène politique avec sa propre formation politique - le Front Démocratique des Francophones (FDF) - et avec ses revendications propres sur le plan politico-linguistique. En conséquence, le fédéralisme belge s'est construit sur un double niveau dès 1980 : le niveau territorial compte trois régions - flamande, wallonne et Bruxelles-Capitale - et le niveau linguistique trois communautés : la Communauté flamande, la Communauté française ${ }^{26}$ et la Communauté germanophone. Tout cela s'est construit au fur et à mesure, avec de nombreux compromis «à la belge».

\section{Pour conclure}

\section{Le néerlandais aujourd'hui}

Aux Pays-Bas, plus de $90 \%$ des habitants connaissent l'anglais, qui est devenu la langue majoritaire de la communication professionnelle dans les grandes entreprises, de l'enseignement supérieur et de la recherche : $23 \%$ de tous les programmes de licence sont entièrement en anglais, et jusqu'à $74 \%$ de tous les programmes de master. Depuis 2019 l'Université Libre d'Amsterdam (VU) a cessé de préparer des étudiants d'ailleurs très peu nombreux - à une licence de néerlandais, tandis que parallèlement, à partir de 2020, l'Université Technique d'Eindhoven n'enseignera plus qu'en anglais.

Il en va différemment en Flandre, où seulement $6 \%$ des programmes de licence et $35 \%$ des programmes de master sont en anglais. Les Flamands, fervents promoteurs du néerlandais, doivent donc constater que leurs voisins du Nord n'y attachent guère d'importance. Les deux

\footnotetext{
26. Il est à noter qu'en Flandre, dès l'origine, le Gouvernement et le Conseil de la Région flamande ont fusionné avec le Gouvernement et le Conseil de la Communauté flamande. En Flandre, il n'y a donc qu'un seul Gouvernement et un seul Conseil.
} 
peuples sont d'ailleurs culturellement fort différents dans les domaines de la vie courante - relations humaines, loisirs, cuisine. Il est certain que les Flamands sont moins attachés aux Pays-Bas que les Wallons à la France. Ils se sont avec peine débarrassés de leur complexe d'infériorité historique par rapport aux francophones, et désormais il semble aussi qu'ils se soient débarrassés d'un complexe d'infériorité linguistique vis-à-vis de leurs voisins du Nord en créant leur tussentaal «langue intermédiaire». En naîtra-t-il une nouvelle variante indépendante de la norme néerlandaise?

\section{Basse-Bretagne et Flandre : deux chemins opposés}

Notre question initiale portait sur l'éventuelle possibilité d'utiliser pour décrire diverses situations sociolinguistiques les outils intellectuels primitivement conçus pour essayer de mieux comprendre le cas particulier de la langue bretonne. Il semble que l'on puisse répondre affirmativement à cette question, à condition de ne pas essayer de saisir «la» langue d'un seul bloc, comme on le fait généralement, ce qui interdit toute comparaison, tant les destins des langues et des sociétés qui les utilisent sont divers et singuliers.

Mais si on distingue les différents niveaux d'usage de ces langues, dans le cadre des institutions sociales au sein desquelles elles sont pratiquées, on obtient alors des objets comparables qui permettent d'extraire la description de la singularité et de jeter des passerelles pardessus les divergences historiques. Ainsi toutes les langues de paysans présentent-elles de nombreux points communs, de même que les langues normées obéissent à des lois plus ou moins semblables.

Des points de similarité existent entre les deux zones que nous avons évoquées : survie tardive des badumes en Basse-Bretagne comme en Flandre, apparition et disparition de plusieurs standards (anversois, brabançon etc. dans la Flandre actuelle, breton dit «de curé» en BasseBretagne jusqu'au milieu du XX siècle), en relation avec l'évolution de diverses institutions; colinguisme avec le français : en des circonstances historiques différentes la Flandre aurait pu se franciser au même titre que toutes les régions de la France moderne. 
Il existe cependant des différences majeures :

- La Flandre dispose de villes anciennes historiquement de langue flamande, dont les institutions marchandes puissantes ont suscité la création de standards adaptés à leurs besoins. En revanche, le développement en Basse-Bretagne des grands ports de guerre de Brest et de Lorient - celui-ci siège, comme son nom l'indique, de la Compagnie des Indes Orientales - sont des créations royales du XVII e siècle et donc de langue française.

- Le breton est isolé, alors qu'au nord de la Flandre se trouve un pays riche possédant une norme linguistiquement proche et solidement établie.

- La Flandre s'est industrialisée au XIX ${ }^{e}$ siècle, et sa bourgeoisie a accompagné son émancipation en adoptant la norme néerlandaise, alors que la Basse-Bretagne ne s'est modernisée que dans la deuxième moitié du $\mathrm{XX}^{\mathrm{e}}$ siècle par le développement industriel de son agriculture.

- La Belgique, unitaire à ses débuts, s'est progressivement régionalisée jusqu’à sa transformation en état fédéral plurilingue. La France, en réaction à la défaite dans la guerre franco-allemande de 1870, a mené une politique résolument tendue vers l'unification linguistique et culturelle du territoire national, préparant les futurs citoyens à l’exercice effectif du français élémentaire ${ }^{27}$. On peut dire que cette politique est parvenue à ses fins : le flamand occidental du Westhoek est, tout comme le breton, en voie d'extinction.

- Une revendication linguistique conservatrice s'est développée dans les mêmes zones, appuyée par l'Église catholique soucieuse de résister à la propagation des idées révolutionnaires, la Flandre y ajoutant la résistance au néerlandais normé vecteur potentiel de la religion protestante. Parallèlement, une tendance nationaliste séparatiste a pris forme dans les deux aires. Elle a pris appui en Flandre sur une bourgeoisie en pleine expansion qui a entraîné le mouvement flamand vers l'adoption de la norme du puissant voisin ; en Basse-Bretagne au contraire, elle n'a trouvé aucun appui institutionnel en dehors d'une frange de la petite bourgeoisie, et aucune norme extérieure sur laquelle pratiques de la langue nationale sous la Révolution française, Paris, Hachette, 1974. 
elle pût s'appuyer en dehors du gallois, langue lointainement apparentée et dont le standard s'était développé au sein des églises protestantes.

Il n'y a aucune prédestination dans le destin de l'une ou l'autre langue. En Flandre, une nouvelle norme semble sur le point de se développer à côté du néerlandais officiel, tandis qu'en Bretagne une norme fictive se répand dans une petite frange de la population sous la tutelle d'un pouvoir régional soucieux de se doter d'une image originale. 